\title{
Current Gas Plasma Sterilization Procedure and Its Future Trends
}

\section{Hideharu Shintani*}

Faculty of Science and Engineering, Chuo University, Tokyo, Japan

Ninety-nine per cent of the material in the universe is in the plasma state. Physicists call plasma the fourth state of matter, after solid, liquid, and gaseous states. Basically, plasma is composed of gas molecules that have been dissociated by an input of energy.

Low-temperature gas plasmas are generated when certain gases are stimulated at atmospheric pressure (AP) or relatively low pressure (LP) with pulsed energy, radio-frequency waves or microwave energy. The plasmas of several different gases such as argon, helium, oxygen, nitrogen or their mixtures have sporicidal activity. Many modern medical devices are thermo- and hydro- sensitive. Given the drawbacks and limitations of other low temperature sterilization procedures, low temperature gas plasma sterilization could represent a useful alternative.

The first practical application of gas plasma sterilization was developed in 1972. Since then several kinds of gas plasma applications have been designed to sterilize the bioburden of various products, and many researchers have studied the subject as well as the mechanism of gas plasma sterilization. The bioburden defines the type and number of viable microorganisms in/on a product (ISO 14161, 11138-1).

Low temperature gas plasmas, used for surface modification and organic cleaning, are ionized gases generated at pressures between 0.1 and 2 torr. These types of plasmas work within a vacuum chamber from which atmospheric gases have been evacuated, typically below 0.1 torr. Low pressure allows for a relatively long free path of accelerated radicals and metastable. Neutral particles such as radicals and metastable can be produced at or near ambient temperatures, and undergo relatively few collisions with molecules at this temperature. Although both radicals and metastable are neutral compounds, their flight distances differ significantly. Whereas the flight distance of a radical is $\sim 0.003 \mathrm{~cm}$ that of a metastable is $\sim 144 \mathrm{~cm}$.

There are three traditional states of matter: liquid gas and solid. Plasma may be considered the fourth state in which the molecules of a gas are excited to become plasma when the gas atoms lose their electrons and generate a highly excited mixture of charged nuclei and free electrons. True plasma is actually considered to consist of positively and negatively charged particles in approximately equal concentrations. Plasma can be generated by the application of sufficient energy, in the form of heat or an electromagnetic field, to a gas. Plasma can be subsequently formed by further energy absorption by the gas, which fragments the gas atoms and molecules to produce negative ions, positive ions, electrons, and other short-lived or long-lived reactive species.

It should be remembered that an atom of any element consists of a central nucleus (made up of positively charged protons and neutrons) that is surrounded by negatively charged and paired electrons, which are organized in defined orbitals depending on their energy levels. In this state, each atom is balanced, with an overall neutral charge produced by an equal number of electrons and protons. As energy is applied to the atoms/molecules in a gas, the molecules and atoms fragment to produce positive ions (as they now have a higher number of protons) and free, negatively charged electrons. In some cases, the electrons react with other atoms, thereby gaining an overall negative charge (negative ions). Further unstable species are also generated including ozone (in the case of oxygen plasmas; ozone causes a serious etching phenomenon) and other free radicals, metastable, photons, UV and VUV. The free radicals that are formed include the hydroxyl radicals $(\mathrm{OH} \cdot)$, NO radicals (NO), oxygen metastable $\left(\mathrm{O}_{2}{ }^{1}\right)$, nitrogen metastable, superoxide anion radical or peroxynitrite anion radical. Free radicals are highly reactive in that they have unpaired electrons in their outermost orbitals and therefore bind with electrons from other molecules to produce a chain reaction of electron loss and gain. Therefore, on exposure to microorganisms, a variety of effects occur, which cause functional damage to cell components (including proteins, lipids, nucleic acids and dipicolinic acid), ultimately resulting in cell death. Furthermore, with the excitation of electrons between atom orbitals, as they return to their natural states, they give off energy $(E=h v)$ in the form of heat or photons, for example, within the UV wavelength range ( 100 to 350 $\mathrm{nm})$. This also contributes to antimicrobial activity as a minor factor. According to experimental results, sterilization effects of UV or VUV on spores are rarely observed, so UV-C is not an efficient contributor to gas plasma sterilization. When the energy source applied to the gas is turned off, the various species rapidly recombine into lower-energy, stable forms.

A variety of gas plasmas can be produced, which are usually named after the gas used to create them, e.g., oxygen or nitrogen gas plasma. Several gases have been used for plasma generation including oxygen, nitrogen, argon, helium, and a mixture of oxygen and nitrogen at $1 / 4(\mathrm{v} / \mathrm{v})$. Most popular is the mixture of oxygen and nitrogen at $1 / 4(\mathrm{v} / \mathrm{v})$. Oxygen alone causes significant etching and shrinkage of microorganisms probably due to ozone as mentioned above. Nitrogen and argon alone do not have significant effects on microorganisms, but the major contributors of nitrogen gas plasma are thought to be metastable of $\mathrm{N}_{2}$ and $\mathrm{O}_{2}$, so the presence of some $\mathrm{O}_{2}$ is favorable; thus $\mathrm{N}_{2} / \mathrm{O}_{2}(4 / 1)$ is the most popular gas for sterilization. As discussed above, plasmas are generated by the application of heat or electromagnetic radiation. Heat is generally not used due to the very high temperatures and pressures required for the generation of plasmas (e.g., up to $3,000^{\circ} \mathrm{C}$ ). Lower-temperature plasmas are usually produced in a gas under vacuum with the application of microwaves or highenergy radiofrequency; in some cases atmospheric pressure plasma is also used, mostly for the purpose of surface modification as well as gas plasma sterilization. These plasmas are usually generated under relatively low pressure such as $1 / 2$ to $1 / 3$ pressures at low temperatures (30 to $\left.50^{\circ} \mathrm{C}\right)$.

According to Shintani, the major contributor of nitrogen gas plasma was confirmed as the peroxynitrite anion radical. Other interesting facts were also clarified by them. In that means, gas plasma sterilization is still under study and several interesting results will be clarified by gas plasma sterilization researchers in a near future.

*Corresponding author: Hideharu Shintani, Faculty of Science and Engineering, Chuo University, 1-13-27, Kasuga, Bunkyo, 112-8551, Tokyo, Japan, Tel: +81425922336; Fax: +81425922336; E-mail: shintani@mail.hinocatv.ne.jp

Received July 20, 2015; Accepted July 21, 2015; Published July 24, 2015

Citation: Shintani H (2015) Current Gas Plasma Sterilization Procedure and Its Future Trends. Pharm Anal Acta 6: e178. doi:10.4172/21532435.1000e178

Copyright: (c) 2015 Shintani H. This is an open-access article distributed under the terms of the Creative Commons Attribution License, which permits unrestricted use, distribution, and reproduction in any medium, provided the original author and source are credited. 